\title{
TITLE:
}

\section{A new design for generation of tunable gamma-ray with a fixed energy electron beam}

$\operatorname{AUTHOR}(\mathrm{S})$ :

Ohgaki, Hideaki; Kii, T.; Toyokawa, H.

\section{CITATION:}

Ohgaki, Hideaki ... [et al]. A new design for generation of tunable gamma-ray with a fixed energy electron beam. IEEE Transactions on Nuclear Science 2009, 56(3): 1316-1320: 5075997.

\section{ISSUE DATE:}

2009-06

URL:

http://hdl.handle.net/2433/84874

\section{RIGHT:}

c 2009 IEEE. Personal use of this material is permitted. However, permission to reprint/republish this material for advertising or promotional purposes or for creating new collective works for resale or redistribution to servers or lists, or to reuse any copyrighted component of this work in other works must be obtained from the IEEE. 


\title{
A New Design for Generation of Tunable Gamma-Ray With a Fixed Energy Electron Beam
}

\author{
H. Ohgaki, T. Kii, and H. Toyokawa, Member, IEEE
}

\begin{abstract}
A tunable quasi-monochromatic gamma-ray beam is indispensable not only for radiation measurements, but also for precise measurements of nuclear data. A design for generation of tunable quasi-monochromatic gamma-ray from the Compton backscattering of a laser light with a fixed energy electron beam has been investigated. We propose a new design (absorber- collimator scheme) by selecting the scattering angle of the Compton backscattered gamma-ray. Tunable gamma-ray can be obtained by changing the solid angle of the collimator (works as a low cut filter) and absorber (works as a high cut filter). A proof of principle experiment has been performed at the AIST-LCS beamline and the result showed that the absorber-collimator scheme could generate tunable gamma-rays. Numerical simulation based on the EGS4 well reproduced the experimental result.
\end{abstract}

Index Terms-Compton backscattering, storage ring, tunable gamma-ray, EGS4.

\section{INTRODUCTION}

A QUASI-monochromatic gamma-ray beam from the Compton backscattering of a laser beam (laser-Compton gamma-ray beam), which collides with a relativistic electron beam, has excellent characteristics, such as a tunable energy, a narrow energy spectrum, and a high polarization [1], [19]. These characteristics are suitable not only for development of the radiation detectors [2], but also for studies on the nuclear physics and the nuclear astrophysics [3]. Moreover, the nuclear resonance fluorescence (NRF) method is considered to have an excellent potential to detect a hidden special nuclear materials [4]. To obtain precise data with a good signal-to-noise ratio in the NRF, a monochromatic tunable gamma-ray beam is indispensable. This is due to the fact that the width of nuclear resonance is quite narrow $(<\mathrm{eV})$ and the overwhelming fraction of gamma-rays outside of the resonance window contributes unwanted background [5].

The energy spread of the laser-Compton gamma-ray beam mostly depends on the quality of the electron beam. Fortunately we can use high energy electron beams whose beam qualities are

Manuscript received June 20, 2008; revised December 26, 2008. Current version published June 10, 2009. This work was supported by the Ministry of Education, Culture, Sports, Science and Technology, Japan, under a Grant-in-Aid for Scientific Research \#19540289.

H. Ohgaki and T. Kii are with the Institute of Advanced Energy, Kyoto University, Gokasho, Uji, Kyoto 6110011, Japan (e-mail: ohgaki@iae. kyoto-u.ac.jp; kii@iae.kyoto-u.ac.jp).

H. Toyokawa is with the National Institute of Advanced Science and Technology, 1-1-1 Umezono, Tsukuba, Ibaraki Japan 305-8568 (e-mail: toyokawa@aist.go.jp).

Color versions of one or more of the figures in this paper are available online at http://ieeexplore.ieee.org.

Digital Object Identifier 10.1109/TNS.2009.2014230 good enough to generate a $1 \%$ energy spread by using storage rings [6]. However, a few number of laser-Compton gamma-ray facilities in MeV-region are operated [6]-[8]. This is due to the fact that most of storage rings are dedicated to the synchrotron radiation experiments and are operated with a fixed energy for stable operations. Therefore, a method for changing the energy of the gamma-ray beam with a fixed energy electron beam is a critical issue. Since a very small fraction of the electron energy is lost in the Compton backscattering process for generation of MeV-region gamma-ray beam, it is quite difficult to apply the tagging method [9], [10]. The other available methods to generate a tunable gamma-ray beam are i) to use a tunable laser, ii) to select a collision angle of the laser-electron interaction, and iii) to choose a scattering angle of the laser-Compton gamma-rays. Numerical studies have been carried out for these three methods [11] and a new design which selects the scattering angles by using set of an absorber and a collimator was proposed.

This paper describes a principle of the absorber-collimator scheme, at first. Then, we will report on the proof of principle experiments and the experimental result will be discussed.

\section{Principle of THE AbSORBer-Collimator Scheme}

The energy of the laser-Compton gamma-ray beam is given by [9]

$$
E_{\gamma}=\frac{\varepsilon_{L}\left(1-\beta \cos \theta_{L}\right)}{1-\beta \cos \theta+\varepsilon_{L}\left(1-\cos \left(\theta_{L}-\theta\right)\right) / E_{e}} .
$$

Here $\varepsilon_{L}$ is the energy of incident laser photon, $E_{e}$ the energy of the electron beam circulating in the storage ring, $\theta$ the gamma-ray scattering angle respecting to the electron beam, $\theta_{L}$ the incident angle of the laser beam. When we tune these four parameters, the energy of the gamma-ray beam can be controlled. To obtain the gamma-ray energy range of $1-10 \mathrm{MeV}$ for the NRF experiment under the condition of the fixed electron energy $\left(E_{e}\right)$ of $1.4 \mathrm{GeV}$, which is a common operational energy of the medium size storage rings, the required wavelength of the laser is calculated to be 3.7-37 $\mu \mathrm{m}$ by using (1). However it is not easy to prepare such tunable laser system. Therefore controlling the incident angle of the laser and/or the scattering angle of the gamma-ray should be studied to generate a tunable gamma-ray beam.

The energy spread of the laser-Compton gamma-ray is written by [9]

$$
\left(\frac{\Delta E_{\gamma}}{E_{\gamma}}\right)^{2} \cong\left(2 \frac{\Delta E_{e}}{E_{e}}\right)^{2}+\left(\gamma \sqrt{\theta_{e}^{2}+\theta_{c}^{2}}\right)^{4}
$$


Here $\Delta E_{e} / E_{e}$ is the energy spread of the electron beam, $\gamma$ the Lorentz factor, $\theta_{e}$ the beam divergence of the electron beam, and $\theta_{c}$ the solid angle of the collimator which determines the scattering angle. One can see from (2) that a high quality electron beam is the crucial point to generate a narrow energy spread of the gamma-ray beam. To obtain a $1 \%$ energy spread of the gamma-ray beam with the beam divergence of the electron beam $\left(\theta_{e}\right)$ of $0.05 \mathrm{mrad}$ and the energy spread $\left(\Delta E_{e} / E_{e}\right)$ of $0.07 \%$, which are typical parameters in medium size storage rings [12], the solid angle of the collimator $\left(\theta_{c}\right)$ is calculated as $0.036 \mathrm{mrad}$. These conditions can be available in a modern storage ring and corresponds to the collimator of $1-\mathrm{mm}$ diameter placed at $14.0-\mathrm{m}$ downstream from the laser-electron collision point in practice. It should be noted that (2) is valid for $\mathrm{CW}$ or long pulse lasers whose bandwidth is negligibly small.

We should also mention the gamma-ray yield per collision, $Y$. From the laser-Compton process $Y$ can be written as [13]

$$
Y=\frac{2 N_{e} N_{L} \sigma L}{A \tau c} .
$$

Here $N_{e}$ is the number of electrons per bunch, $N_{L}$ the number of laser photons per pulse, $\sigma$ the integrated cross section within the collimator solid angle, $L$ the effective length where the collision takes place, $A$ the cross sectional area of the larger beam, $c$ the speed of light, and $\tau$ is the pulse width of the longer beam. It is clear from (3) that the incline collision scheme $\left(\theta_{L}\right)$ with a long pulse laser decreases the photon yield because the effective length, $L$, is as small as the electron beam size $(<\mathrm{mm})$. Equation (3) also indicates us that a small electron beam size is preferable to generate an intense gamma-ray beam, because the cross sectional area, $A$, is proportional to square of the electron beam size. Therefore, to obtain an intense gamma-ray beam with the inclined collision scheme requires a short pulse laser. Such scheme has already been demonstrated by R. W. Schoenlein [14]. However, this method uses the short pulse mode-lock laser system with a precise timing-stabilization system to compensate the timing jitters of the laser pulse with respect to that of the electron bunch. In general, these laser system have small average powers comparing to a long pulse or $\mathrm{CW}$ lasers. Because we needed a high-average gamma-ray yield for a practical use, we have concentrated on using a long pulse or $\mathrm{CW}$ laser system in this study.

Therefore, controlling the gamma-ray scattering angle $(\theta)$ is the other considerable method. In practice, we can generate tunable gamma-ray beam with a collimator which is placed in the off-axis scattering angle. However, the gamma-ray yield is severely decreased with this method. Therefore, as is shown in Fig. 1, we propose a new technique using a collimator, which cuts the low energy gamma-rays, and an absorber put on the $\theta=0$ axis, which stops the high energy gamma-rays.

For instance, to select $10-\mathrm{MeV}$ gamma-ray with $1.064 \mu \mathrm{m}$ laser, the scattering angle is $0.576 \mathrm{mrad}$ for $1.4 \mathrm{GeV}$ electron beam. To obtain a $1 \%$ energy spread of gamma-ray beam $\left(E_{\gamma}=\right.$ $9.90 \mathrm{MeV}$ ) the scattering angle can be calculated to be 0.580 mrad. Thus, if we put a collimator of $20 \mathrm{~mm}$ in diameter at 17.241-m downstream, the collimator works as a low energy filter. On the other hand, if we put an absorber whose diameter is $20 \mathrm{~mm}$ at $17.366-\mathrm{m}$ downstream, the absorber works as a

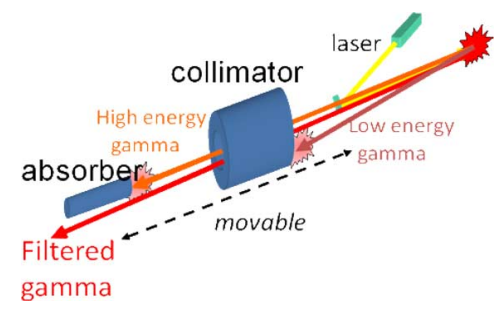

Fig. 1. Principle of the absorber-collimator method.

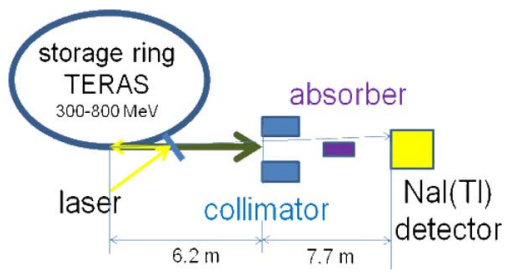

Fig. 2. Schematic drawing of the experimental set-up.

high energy filter. Since the distance control is more precise and easier than changing the aperture size of the collimator and/or the absorber, we can tune the gamma-ray energy by moving the collimator and the absorber position while keeping their diameters in constant in this study. By using this system the gamma-ray flux is expected to be order of $10^{5}$ photons $/ \mathrm{s} / 100$ $\mathrm{mA}$ for 10-W Nd:YAG laser [11].

We should note that the polarization of the gamma-ray beam will be degraded by this system, because large scattering angle leads depolarization in the scattered gamma-ray in Compton process. Therefore the spin-parity measurement [15] would be difficult with this system. We plan to apply the gamma-ray beam to nuclear physics study and nuclear data evaluation that require many gamma-ray photons exposed onto a target material integrated over measurement time. Because the beam is exposed onto a target which is a few $\mathrm{cm}$ in diameter in typical dimension, the beam can be circular or ring-shaped ones. With this apparatus, however, the energy tunability plays an important and critical role as we reported in this study. In addition, the ring shaped gamma-ray beam can provide a large irradiation area which could be useful for detection of hidden special nuclear materials.

\section{PROOF OF PRINCIPLE EXPERIMENT}

The proof of principle experiment was conducted in the AIST-LCS beamline [16], as shown in Fig. 2. The energy of the electron beam was $600 \mathrm{MeV}$ and the beam current was $100 \mathrm{~mA}$. A Nd:YAG laser $(\lambda=1064 \mathrm{~nm})$ of $1-\mathrm{W}$ output power was used. A lead block collimator of $8 \mathrm{~mm} \phi$ whose length was $20 \mathrm{~cm}$ was placed at $6.2 \mathrm{~m}$ in the downstream of the laser-electron interaction region. Therefore, the top energy of $6.35 \mathrm{MeV}$ can be derived by (1) and the energy spread of $36 \%$ (in full width) derived by (2). Here we assumed that the solid angle of the collimator defined the energy spread. An $8 \mathrm{~mm} \phi$ lead absorber of $10 \mathrm{~cm}$ in length was set at $\theta=0$ position. We put the absorber on an optical rail which was placed behind the collimator for precise movement of the absorber position along the downstream of the gamma-ray beamline. The energy spectrum and the yield of the gamma-ray beam were measured 


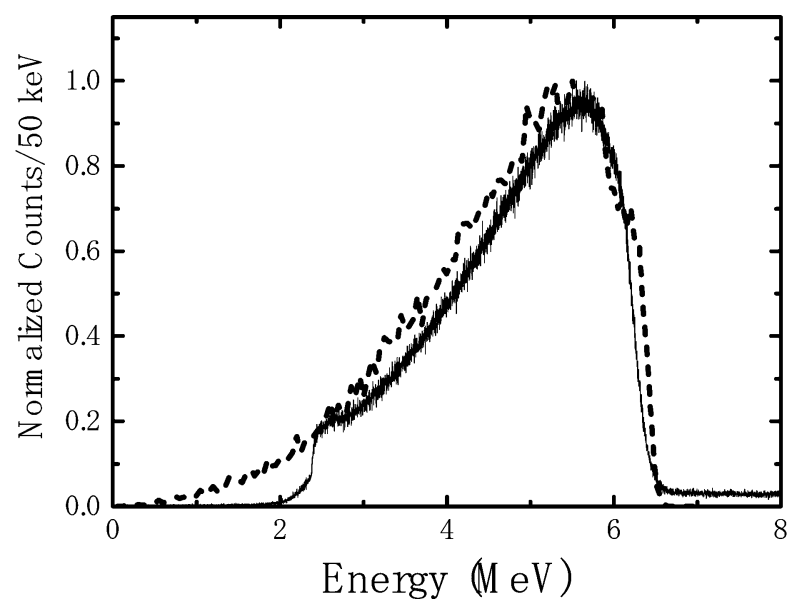

Fig. 3. Energy spectrum of the gamma-rays with the $8-\mathrm{mm} \phi$ collimator. Dotted line shows the result of the EGS4 calculation.

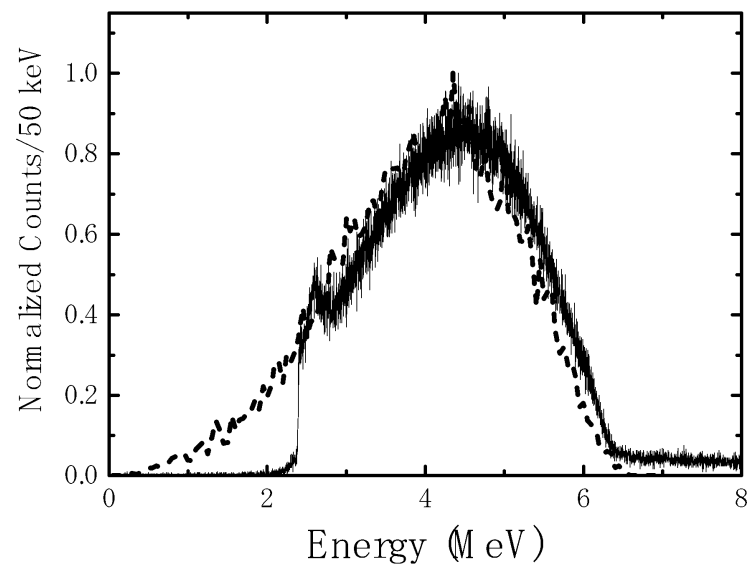

Fig. 4. Energy spectrum of the gamma-rays with the $8-\mathrm{mm} \phi$ collimator and the 8-mm $\phi$ absorber placed at 370-cm downstream from the collimator. Dotted line shows the result of the EGS4 calculation.

by using a $\mathrm{NaI}(\mathrm{Tl})$ detector of $8^{\prime} \phi \times 10^{\prime}$ which was located at $7.7-\mathrm{m}$ behind the collimator. The energy resolution of the detector was $8 \%$ for ${ }^{137} \mathrm{Cs}$.

The measured energy spectrum with the collimator only is shown in Fig. 3. The spectrum observed shows a typical laserCompton gamma-ray beam, the sharp drop in the high energy region (around $6.3 \mathrm{MeV}$ ) and the gradual decrease in the low energy region. The peak energy of the gamma-ray was $5.7 \mathrm{MeV}$ and the energy spread was $15 \%$ (rms). A monte-carlo calculation with EGS4 [17] was also shown in Fig. 3 as the dotted-line. The detector resolution, the electron beam size and the effective length of the laser-electron interaction region were taken into account the calculation. One can see a good agreement between the observation and the calculation.

The energy spectrum with the absorber-collimator configuration was shown in Fig. 4. The absorber was located at $370 \mathrm{~cm}$ behind the collimator. The cut-off energy in high energy region was analytically predicted as $5.21 \mathrm{MeV}$ by (1). Therefore, we can expect $22 \%$ energy spread of the gamma-ray beam $(\sim 9 \%$ in rms). As is shown in the figure, the peak energy was clearly moved toward the lower energy than that without the absorber (Fig. 3). The peak energy was moved from $5.7 \mathrm{MeV}$ to $4.5 \mathrm{MeV}$.

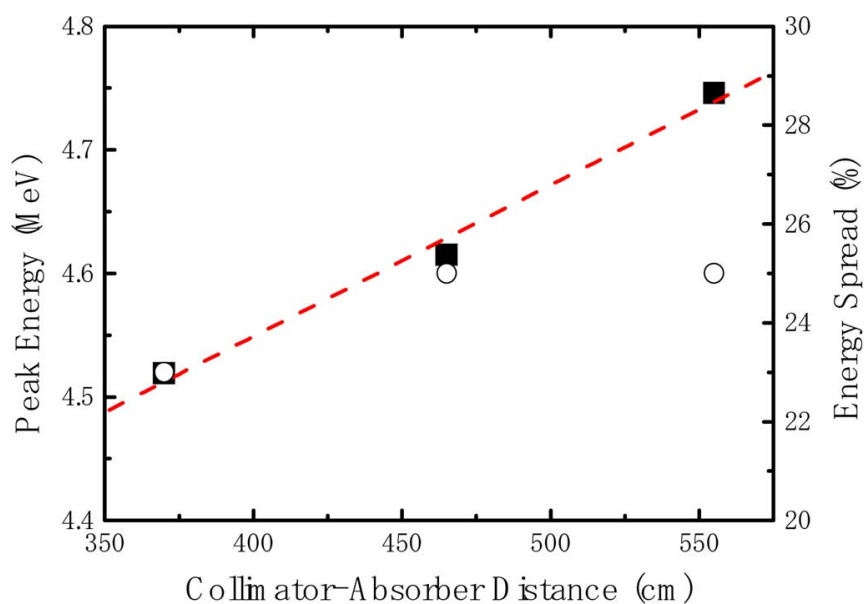

Fig. 5. Gamma-ray energy as a function of th collimator-absorber distance (square dots). The broken-line shows an eye-guide. Energy spread of the gamma-ray also plotted as open circles.

Although the peak energy of the gamma-rays was successfully tuned by the absorber-collimator scheme, the energy spread was more than $20 \%$ (rms) which was broader than the simple analytic expectation. We also carried out the EGS4 simulation. The result was also plotted as the dotted-line in Fig. 4. As is shown in Fig. 4 the EGS4 calculation successfully reproduced the experimental result.

To check the energy tunability, the absorber-collimator distance was changed from $370 \mathrm{~cm}$ to $470 \mathrm{~cm}$, and to $570 \mathrm{~cm}$. The cut-off energies were expected as $5.36 \mathrm{MeV}$ at $470 \mathrm{~cm}$ and $5.50 \mathrm{MeV}$ at $570 \mathrm{~cm}$. Since we fixed the collimator position and moved the absorber only, the absorber-collimator distance was also changed the energy spread of the gamma-rays as $10 \%(\mathrm{rms})$ for $470 \mathrm{~cm}$ and $11 \%(\mathrm{rms})$ for $570 \mathrm{~cm}$. Fig. 5 shows the result of the energy (square dots) and the energy spread (open circles) of the gamma-ray beam as a function of the distance between the collimator and the absorber. It should be noted that the energy of the gamma-ray beam was successfully tuned by the distance between the collimator and the absorber, but the energy spread was insensible to the distance. We will discuss on the energy spread in the next section.

The gamma-ray yield as a function of the absorber solid angle is shown in Fig. 6. As is shown in Fig. 6, a linear relation is clearly observed between the gamma-ray yield and the absorber solid angle. The gamma-ray yield of order of $10^{4}$ with $1-\mathrm{W}$ laser was obtained by the absorber-collimator scheme. It could be noted that the gamma-ray yield of more than $10^{5}$ could be obtained by using a $10-\mathrm{W}$ Nd:YAG laser. This yield would be acceptable to perform the NRF experiment [18].

\section{DisCUSSION AND CONCLUSION}

In previous section the measured energy spread of the gamma-ray beam shows very large one $>20 \%$ and was insensible to the collimator-absorber distance. One can notice that the sharp drop in the high energy region in Fig. 3 disappears in Fig. 4 and turns to the gradual slope which is very similar to the low energy one. This could be explained that the finite length of the laser-electron interaction region broadened the absorber solid angle. We used the laser optics system with the Rayleigh 


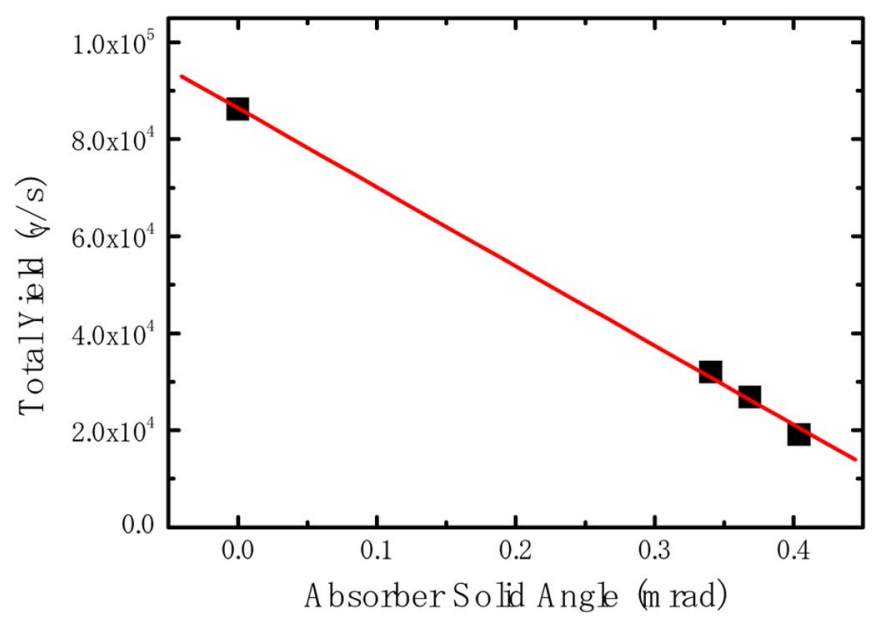

Fig. 6. Yield of the gamma-ray as a function of the solid angle of the absorber.

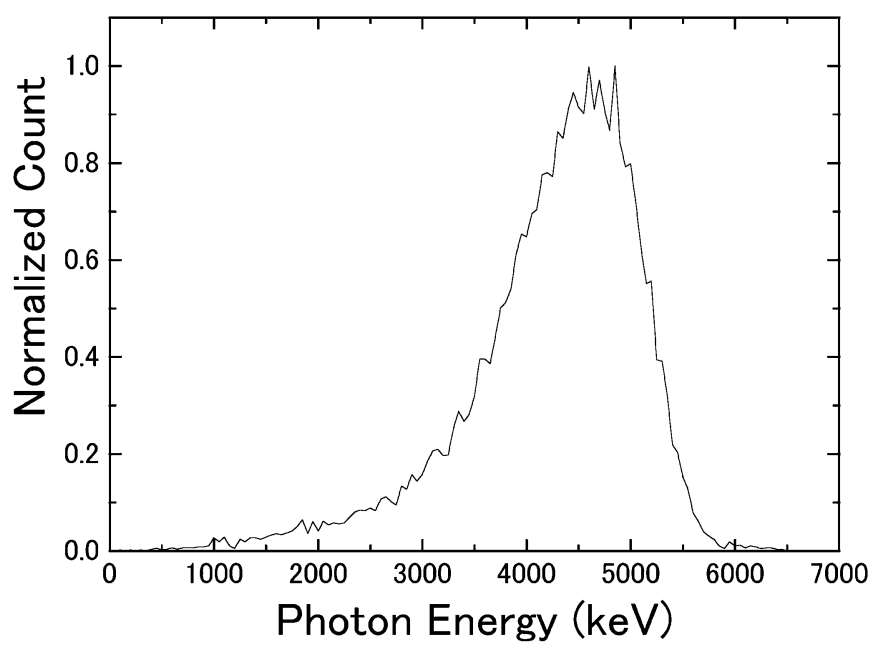

Fig. 7. Energy spectrum of the gamma-ray beam with EGS4 calculation assuming the beam size of the electron beam of $0.5 \mathrm{~mm}$.

length of $\sim 50 \mathrm{~cm}$ which corresponds to the effective interaction length and could not neglect in comparison with the distance between the absorber and the laser-electron interaction region. As a result, about $8 \%$ fluctuation is calculated in the absorber solid angle. On the other hand, the sharp drop in the Fig. 3 is due to the 180 degree scattered gamma-rays which is maximum energy of the Compton backscattering (1). Therefore, there is no chance to broaden the energy spectrum over this sharp drop except for the energy spread of the electron beam which is a very small value, $\Delta \mathrm{E} / \mathrm{E} \sim 10^{-4}$, in the storage ring.

Moreover, the fact that the measured energy spread was insensible to the collimator-absorber distance suggests that the energy spread was not determined by the geometric condition only. A possible explanation is the large electron beam size $(\sim 2 \mathrm{~mm})$ in this experiment. The previous numerical study predicted that larger beam size of the electron beam made broader energy spectrum [11]. To confirm this situation, we performed the simulation with a small electron beam size of $0.5 \mathrm{~mm}$. Fig. 7 shows the result of the simulation. It is clearly shown that the electron beam size of $0.5 \mathrm{~mm}$ generates a narrower energy spread (13\%) of the gamma-ray beam.
We should note that the present simulation also includes the detector resolution which was good enough for the present energy spread of the gamma-ray beam. Thus, in order to obtain further narrow bandwidth of the gamma-ray beam, an electron beam of small emittance and a pulse laser of ps duration are required. Simulations predicted that the electron beam of 25 nmrad emittance and $10 \mathrm{ps}$ laser pulse could produce about $7 \%$ gamma-ray beam by using the Absorber-Collimator scheme.

Consequently, a new design (Absorber-Collimator scheme) to generate tunable gamma-ray beam in $\mathrm{MeV}$ region was proposed. A proof of principle experiment has been carried out at the AIST-LCS beamline. By using an $8-\mathrm{mm} \phi$ collimator, which was placed at $6.2-\mathrm{m}$ downstream of the electron-laser interaction point, and an $8-\mathrm{mm} \phi$ absorber, which was placed at 370 $\mathrm{cm}$ behind the collimator, the energy of gamma-ray was successfully tuned from $5.7 \mathrm{MeV}$ to $4.5 \mathrm{MeV}$. On the other hand the measured energy spread was broad one $(>20 \%)$ and insensible to the absorber solid angle. This broad energy spread was due to the effective length of the laser-electron interaction region and to the large electron beam size of $2 \mathrm{~mm}$. An EGS4 calculation which included the detector response, the effective length of the laser-electron interaction region, and the electron beam size well reproduced the measured energy spectrum.

The yield of the gamma-ray was $10^{4} \mathrm{\gamma} / \mathrm{s} / 100 \mathrm{~mA} / 20 \% \mathrm{BW} / \mathrm{W}$ and showed a linear dependence of the absorber solid angle. The total gamma-ray flux of $>10^{5} \mathrm{\gamma} / \mathrm{s} / 100 \mathrm{~mA}$, which is large enough for the NRF experiment, can be obtained with a $10-\mathrm{W}$ laser.

\section{ACKNOWLEDGMENT}

H. Ohgaki thanks Y. Uozumi and M. Imamura (Kyushu Univ.), T. Shizuma and T. Hayakawa (JAEA) for their help with the AIST experiment and for valuable discussions on application of the tunable gamma-rays. He also thanks S. Koda, Y. Iwasaki, and Y. Takabayashi (SAGA-LS) for discussing the beam quality of the medium size storage ring.

\section{REFERENCES}

[1] R. H. Milburn, "Electron scattering by an intense polarized photon field," Phys. Rev. Lett., vol. 10, p. 75, 1963.

[2] K. Kudo, N. Takeda, S. Koshikawa, H. Toyokawa, H. Ohgaki, and M. Matzke, "Photon spectrometry in thermal neutron standard field," Nucl. Instrum. Methods Phys. Res. A, vol. 476, no. 1, 2, pp. 213-217, 2002.

[3] H. Utsunomiya et al., "Cross section measurements of the ${ }^{181} \mathrm{Ta}(\gamma, \mathbf{n})$ ${ }^{180}$ Ta reaction near neutron threshold and p-process nucleosynthesis," Phys. Rev. C, vol. 67, no. 1, pp. 158071-158079, 2003.

[4] W. Bertozzi, S. E. Korbly, R. J. Ledoux, and W. Park, "Nuclear resonance fluorescence and effective $\mathrm{Z}$ determination applied to detection and imaging of special nuclear material, explosives, toxic substances and contraband," Nucl. Instr. Meth. Phys. Res. B, vol. 261, pp. 331-336, 2007.

[5] J. Pruet, D. P. McNabb, C. A. Hagmann, F. V. Hartemann, and C. P. J. Barty, "Detecting clandestine material with nuclear resonance fluorescence," J. Appl. Phys., vol. 99, p. 123102, 2006.

[6] V. N. Litvinenko, "Recent results with the high intensity $\gamma$-ray facility," Nucl. Instr. Methods A, vol. 507, no. 1-2, pp. 527-536, 2003, For HI $\gamma$ S, MEGA Collaborations.

[7] H. Ohgaki, T. Noguchi, S. Sugiyama, T. Mikado, M. Chiwaki, K. Yamada, R. Suzuki, N. Sei, T. Ohdaira, and T. Yamazaki, "Polarized gamma-rays with laser-Compton backscattring," Nucl. Instrum. Methods Phys. Res. A, vol. A375, no. 1, pp. 602-605, 1996.

[8] K. Aoki et al., "High-energy photon beam production with laser-Compton backscattering," Nucl. Instr. and Meth. A, vol. 516, p. $228,2004$. 
[9] A. M. Sandorfi et al., "High energy gamma ray beams from compton backscattered laser light," IEEE Trans. Nucl. Sci., vol. NS-30, pp. 3083-3087, 1983.

[10] T. Nakano et al., "Multi-GeV laser-electron photon project at SPring-8," Nucl. Phys. A, vol. 684, pp. 71-79, 2001.

[11] H. Ohgaki, S. Koda, Y. Iwasaki, Y. Takabayashi, K. Yoshida, T. Tomimasu, Y. Uozumi, and K. Ishibashi, "Study on energy variable lasercompton gamma-ray with a fixed energy electron beam," J. Nucl. Sci. Technol., vol. 44, no. 5, pp. 698-702, 2007.

[12] T. Tomimasu et al., "Commissioning of SAGA light source," in Proc. PAC2005, Knoxville, TN, May 16-20, 2005, pp. 4021-4023.

[13] C. K. Sinclair, J. J. Murray, P. Klein, and M. Rabin, "A polarized photon beam for the SLAC 82-inch hydrogen bubble chamber," IEEE Trans. Nucl. Sci., vol. NS-16, pp. 1065-1068, 1969.

[14] R. W. Schoenlein, W. P. Leemans, A. H. Chin, P. Volfbeyn, T. E. Glover, P. Balling, M. Zolotorev, K.-J. Kim, S. Chattopadhyay, and C. V. Shank, Science, vol. 274, p. 236, 1996.
[15] N. Pietralla, Z. Berant, V. N. Litvinenko, S. Hartman, F. F. Mikhailov, I. V. Pinayev, G. Swift, M. W. Ahmed, J. H. Kelley, S. O. Nelson, R. Prior, K. Sbourov, A. P. Tonchev, and H. R. Weller, "Parity measurements of nuclear levels using a free-electron-laser generated $\gamma$-ray beam," Phys. Rev. Lett., vol. 88, p. 012502, 2002.

[16] H. Toyokawa, T. Mikado, K. Yamada, and H. Ohgaki, "High-energy photon radiography system using laser-Compton scattering for inspection of bulk materials," Rev. Sci. Instrum., vol. 73, no. 9, pp. 3358-3362, 2002.

[17] W. R. Nelson, H. Hirayama, and D. W. O. Roger, "The EGS4 code system," in SLAC-265, 1985.

[18] H. Ohgaki et al., "Generation and application of laser-Compton gamma-ray at ETL," Nucl. Instr. Meth. A, vol. 455, pp. 54-59, 2000.

[19] F. R. Arutyunian and V. A. Tumanian, "The Compton effect on relativistic electrons and the possibility of obtaining high energy beams," Phys. Lett., vol. 4, p. 176, 1963. 